\title{
Enhanced Resource Reservation Technique for Reduced Call Blocking in Femtocell Networks
}

\author{
Amit Kumar Vishwakarma, Akhilesh Jain, Swapnil Jain \\ NRI Institute of Information Science \& Technology \\ Dept. of Electronics and Communication \\ Bhopal, Madhya Pradesh, India
}

\begin{abstract}
Femtocell has proved to be a promising technology to enhance indoor coverage and network throughput. Dense deployment of femtocells facilitates efficient offloading of data traffic from the macrocell network to the femtocell network. However, this dense deployment may result in serious inter-femtocell interference. Considering limited coverage radius of the femtocell, frequent handovers is another challenge which may result in excessive call dropping. In order to improve QoS of mobiles users in terms of call blocking ratio, we suggest a predictive resource reservation technique. Our proposed technique first calculates the probability distribution of UEs' locations in order to optimally deploy femtocells using Active LeZi Algorithm. Then, we use regression-based prediction algorithm (Box-Jenkins Model) to forecast the inter-call arrival and call duration distribution. Finally, we reserve the resources for users in multiple femtocells as to reduce the chances of call dropping. Our proposed technique has shown significantly improve the performance of the network in terms of call blocking, system throughput, and energy efficiency.
\end{abstract}

\section{Keywords}

Femtocell, Resource Reservation, Throughput, Energy Efficiency

\section{INTRODUCTION}

Future wireless networks require ubiquitous connectivity with high data rates to improve the quality of service (QoS) of mobile users. To handle exponential increasing demands for high bitrate and ubiquitous connectivity, mobile operators are looking for dense deployment of miniature cellular base stations inside users' homes/offices. Deployments of such low-cost, low-powered cellular base stations at close proximity to mobile users not only improve network throughput, coverage, and reliability but also enhance the life of battery-operated mobile devices. These small base stations are more commonly known as Femtocell or Femto Access Points (FAPs) or simply Femtocells.

Dense deployment of FAPs allows efficient reuse of available wireless spectrum. This leads to improvement in the system capacity manifold without increasing additional deployment and maintenance costs to the cellular operators. Additionally, it also helps in solving indoor coverage problem as indoor users are severely affected by wall penetration losses. The unique benefit of femtocell technology is that users require no new equipment. Any existing mobile user can connect to and get serviced by FAPs. Additionally, the deployment and maintenance costs of the femtocell are very low which make it feasible for both home users and cellular operators. Indeed, a well-designed femtocell/macrocell-integrated network can divert huge amounts of traffic from congested and expensive macro-cellular networks to femto-cellular networks. From the wireless operator point of view, the ability to offload a large amount of traffic from macro-cellular networks to femtocells is the most important advantage of the femtocell/macrocell-integrated network architecture [1]. This will not only reduce the investment capital, maintenance expenses, and operational costs but will also improve the reliability and energy efficiency of the cellular networks [2]. It is observed that the count of femtocell deployment all over the world already exceeded the total macrocell count. The count is expected to further increase in future as operators are now looking for cost effective coverage extension in rural and remote femtocell deployments. This will result in increased co-tier interference to neighboring femtocell users. Additionally, with high data demands from indoor users, it might not be possible for femtocells to always serve incoming mobile users' request. In order to avoid this, prior resource reservation is suggested in the literature. Authors in [3] have suggested radio resource reservation at the future locations based on users' mobile trajectory. Authors in [4] proposed a fair scheduling algorithm combined with reservation resource for supporting differentiate application-level services. A unique resource management scheme that contains bandwidth adaptation policy and dynamic bandwidth reservation policy is proposed in [5].

Our work focuses on using Box-Jenkins algorithm to perform forecast of inter-call arrival and call duration. Box- Jenkins algorithm has already been used for electricity load and price forecasting [6] 7]. Additionally, ARIMA models based on Box-Jenkins methodology for capturing the self-similar internet traffic is done in [8]. Authors in [9] proposed a model-based bandwidth prediction scheme for variable-bit-rate (VBR) video traffic with regular group of pictures (GOP) pattern. Multiplicative ARIMA (autoregressive integrated moving-average) is used as a base stochastic model for prediction and model validity check.

In this paper, we use statistics based approach to calculate the probability of occurrence of mobile users in home/office environment. Based on this probability distribution, we optimally deploy femtocells inside rooms whilst considering penetration ratio; which is the fraction of rooms in which femtocell are deployed. Then, we learn the mobile users mobility model and perform femtocell detection as 
to find out the possible candidate femtocells that a mobile user can move to. Finally, resource reservation in done in those candidate femtocells for a finite duration as to reduce mobile users' blocking during handovers.

The rest of the paper is organized as follows. Section 2 explains the model of femtocell network along with 2D apartment structure, user mobility model, and cell selection scheme. Additionally, channel model, energy consumption, and energy efficiency parameters are also discussed. In section 3 we formulated our problem and presents our proposed resource reservation technique based on ARIMA model. Section 4 presents the simulation scenario and obtained results. Finally, we conclude our work in section 5

\section{SYSTEM MODEL}

Our system model consists of a group of Femtocell or Femto Access Points (FAPs) deployed in the simulation region. User Equipments (UEs) are deployed in such a way that each UE can connect to at least one FAP. FAPs are assumed to be in "open Access" mode and can serve any UEs in their vicinity. Only downlink data transmission is investigated in our analysis. Total available bandwidth is divided into $N$ subchannels each $\mathcal{W}$ hertz wide. We consider OFDMA and Rayleigh flat fading to render all subchannels identically over long run [10]. A total of $U$ UEs are uniformly deployed inside rooms while FAPs are placed at the center of the room. The count of total deployed FAPs depends upon the penetration ratio $\left(P_{r}\right)$. Additionally, placement locations for FAPs are calculated based on the cumulative probability distribution of UEs in different rooms; which is explained in Subsection 2.2 Inter-call arrival and call duration of UEs follow an exponential distribution.

\subsection{Apartment Structure Model}

we consider a single floor apartment with $R$ rectangular rooms of different sizes as shown in Figure 1 . UEs can move within the apartment, one room at a time according to Modified Random Waypoint Mobility Model explained in next subsection. The connectivity among various rooms is represented using a graph $G$ where vertices represent the rooms and existence of an edge represents the connectivity between a pair of rooms. For example, Figure 1 represents a 5 room apartment on the left and respective connectivity between rooms using a graph on the right.
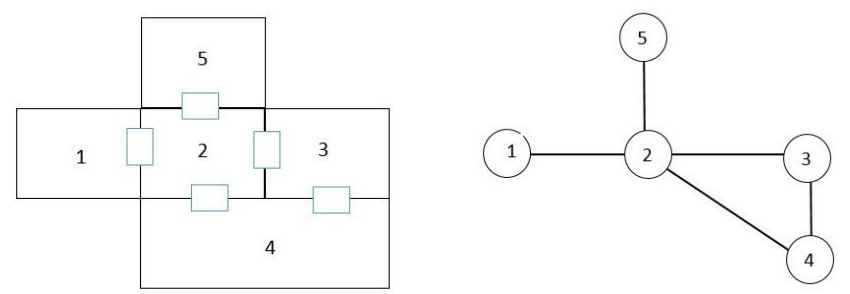

Fig. 1. Apartment Structure

\subsection{UE Mobility Model}

The UE mobility model considered for our analysis is Random Waypoint Mobility Model with a few modifications. UEs can move randomly and freely within the apartment with a restriction that UEs can move from one room to another only if they are connected.
However, the destination, speed, and direction are all chosen randomly and independently of other UEs. The movement of UEs is governed in the following manner: Each UE selects a random destination room in the simulation area based on the connectivity graph $G$ and a random speed between 0 and $S_{\max }$. Once, UE moves to this destination, it pauses for a fixed time period before selecting another random location and speed for movement. This behavior is repeated for the entire simulation period.

\subsection{Cell Selection Scheme: Max RSRP}

RSRP is defined as the linear average of the downlink reference signals (RS) across the entire channel bandwidth. This scheme considers Reference Signal Received Power (RSRP) based association for UEs. At the time of cell selection, UEs get associated with the base station (BS) providing highest RSRP. So, the $i^{t h} \mathrm{UE}$ will select the $k^{\text {th }} \mathrm{BS}$ as its serving $\mathrm{BS} i$ if,

$$
\text { CellID } D_{i}=\arg _{k} \max \left(R S R P_{k}\right)
$$

Knowledge of absolute RSRP provides the UE with essential information about the signal strength of cells from which path loss can be calculated. It is one of the fundamental UE downlink physical layer measurements, and is mostly utilized during the decision making in handovers.

\subsection{Channel Model and Variable Bit-rate Transmission}

Let $P_{t x, f}$ represents the instantaneous subchannel transmit power of FAP $f$. Considering Reuse 1 scheme among all FAPs, the average downlink (DL) SINR of UE $i$ when connected to FAP $f$ is given by,

$$
\Gamma_{i, f}=\frac{P_{t x, f} G_{i}^{f}}{\sum_{l=1, l \neq f}^{\mathcal{F}} P_{t x, l} H_{i}^{l}+\mathcal{W} N_{0}}
$$

where $G_{i}^{j}\left(H_{i}^{j}\right)$ is the effective signal (interference) gain to UE $i$ from FAP $j . N_{0}$ is the noise spectral density.

Based on this DL SINR, the average bitrate obtained by UE $i$ from FAP $f$ is calculated by Shannon Hartley theorem as [11],

$$
B_{i, f}=N_{i, f} * \mathcal{W} * \log _{2}\left(1+\Gamma_{i, f}\right) \quad \text { bits } / s
$$

where $N_{i, f}$ is the number of subchannels allocated to user $i$ from FAP $f$. Here, we consider that a UE can associate with only one FAP at any point of time. Hence, the following constraint is enforced for each FAP $f \in \mathcal{F}$,

$$
\sum_{i=1}^{U} N_{i, f} \leq N
$$

\subsection{Energy Consumption and Energy Efficiency}

Energy consumption of FAPs is taken to be load dependent with some fixed "Zero Load" consumption. The total energy consumption of a FAP can be calculated using the following equation [12],

$$
E_{F A P}=E_{0}+\left(\frac{T_{m}}{\varsigma_{P A}}+P_{S P}\right)
$$

where $E_{0}$ is fixed "Zero Load" power. $\varsigma_{P A}$, and $P_{S P}$ represent power amplifier efficiency and signal processing overhead, respectively. Here $T_{m}$ is total input power to transmitting antenna ob- 
tained by summing up transmit power $\left(P_{t x, f}\right)$ of all the subchannels in use. For each FAP, this $P_{t x, f}$ can take value between $\left(P_{\text {min }}\right.$ and $P_{\max }$ ).

To evaluate energy efficiency, we compute the ratio of total consumed power to total system throughput. This ratio is called Energy Consumption Rating (ECR), and denotes energy consumption normalized to throughput (Watts/Mbps) [13].

$$
E C R=\frac{\text { Energy Consumption }}{\text { Effective System throughput }}
$$

Hence, lower the value of ECR, more energy efficient the system is.

\section{PROBLEM FORMULATION \& SOLUTION DESCRIPTION}

Our problem focuses on improving QoS of UEs in terms of call blocking probability. Since coverage radius of FAP is small, UEs may move out of its connected FAP during a call. Hence, an efficient handover mechanism is necessary to continue the UE's call. Following are the objective of our work:

- Statistical and Markov model based UE location analysis for optimal placement of FAPs inside apartment rooms considering penetration ratio.

- Statistics based FAP detection inside various rooms.

- Learning based UEs' inter-call arrival and call duration estimation.

-Efficient resource reservation for UEs in neighboring femtocell to avoid call blocking during handovers.

-Analysing system throughput and energy efficiency aspects of suggested resource reservation technique.

\subsection{UE Location Statistics and Optimal FAP Placement}

Dense deployment of FAPs helps in improving the throughput of a cellular network by efficient reuse of wireless spectrum. However, it may result in high co-channel interference. Hence, FAPs count and deployment locations should be controlled in order to maximize coverage while minimizing interference and costs. We consider an adaptive probability-based strategy to first analyze UEs location during entire simulation. For this, we maintain a 2D matrix $\mathcal{U}$ of size $U \times R$ which contains the count of each UE occurrences in all the rooms (Fig. 4). Hence, whenever UE $i$ moves to room $j$, it increments the value of $\mathcal{U}[i][j]$. Now, for optimal FAPs deployment, we present ActiveLeZi prediction algorithm [12]. This algorithm is based on the $L Z 78$ data compression algorithm, which incrementally gathers the information and delivers the real-time online predictions. For this, we closely follow the approach of authors discussed in [14]. An input sequence is provided and as the number of states in that input sequence grows, the loss of information across the phase boundaries also increases rapidly. For this, we suggest a solution that maintains a variable length window of previously seen symbols. The length of the window at each stage is taken equal to the length of the longest phrase encountered. Within this window, statistics on all possible contexts is gathered. This helps in building a better approximation to the order-k Markov model because it has captured information about contexts in the input sequence that cross phrase boundaries in the classical LZ78 parsing. Hence, it results in a better convergence rate to optimality predictability and a greater predictive accuracy. Figure 3 illustrates the algorithm

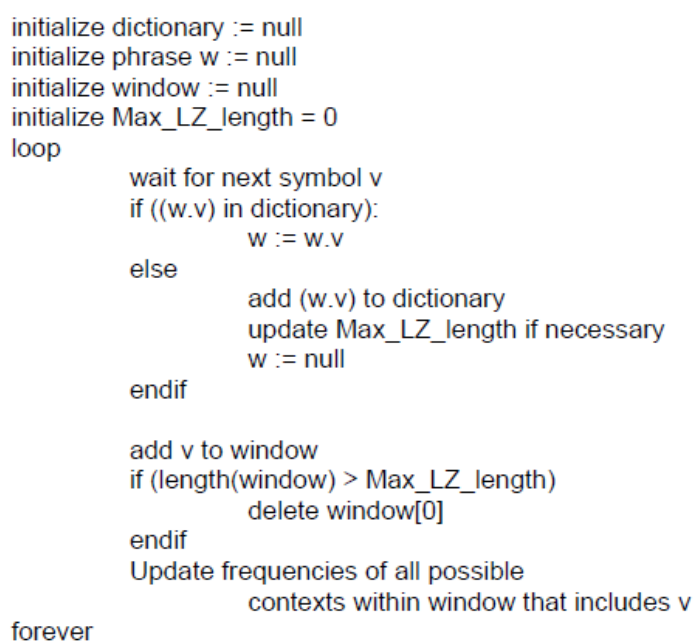

Fig. 2. Active Lezi algorithm.

represents the pseudocode of Active LeZi algorithm with considerable details. Figure 4 represents the trie formed by the Active LeZi parsing of the input sequence "aaababbbbbaabccddcbaaaa".

We calculate the probability of each state occurring in the sequence and predict the one with the highest probability as the most likely next action. This is done by predicting the next event of the sequence for which ALZ has built a model. Then, we use data compression and Prediction by Partial Match (PPM) family of predictors symbol. In our example, the last phrase aaa (which is also the current ALZ window) is used.

Within this phrase, the contexts that can be used are all suffixes within the phrase, except the window itself (i.e. aa, a, and the null context). Suppose the probability that the next symbol is an "a" is being computed. From Figure 4 we see that an "a" occurs two out of the five times that the context aa appears, the other cases producing two null outcomes and one $b$. Therefore, the probability of encountering an "a" at the context aa is $2 / 5$, and we now fall back to the order- 1 context (i.e. the next lower order model) with probability $2 / 5$. At the order- 1 context, we see an "a" five out of the ten times that we see the "a" context, and of the remaining cases, we see two null outcomes. Therefore, we predict the "a" at the order-1 context with probability $5 / 10$ and escape to the order- 0 model with probability $2 / 10$. At the order 0 model, we see that 10 out of 23 symbols seen so far, and we, therefore, predict a with probability $10 / 23$ at the null context. The blended probability of seeing an "a" as the next symbol is, therefore:

$$
\frac{2}{5}+\frac{2}{5}\left\{\frac{5}{10}+\frac{2}{10}\left(\frac{10}{23}\right)\right\}
$$

This method of assigning probabilities has the following advantages:

-It solves the zero-frequency problem.In the above example, if only the longest context had been chosen to make a decision on probability, it would have returned a zero probability for the symbol "c", lower-order models show that this probability is indeed non-zero.

- This blending strategy assigns greater weight to higher-order models in calculating probability if the symbol being considered 


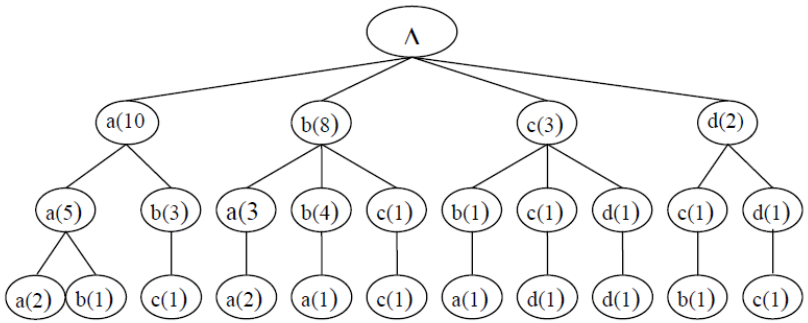

Fig. 3. Trie formed by the ALZ parsing of the string "aaababbbbbaabccddcbaaaa"

is found in that context while lower-order models are suppressed owing to the null context escape probability.

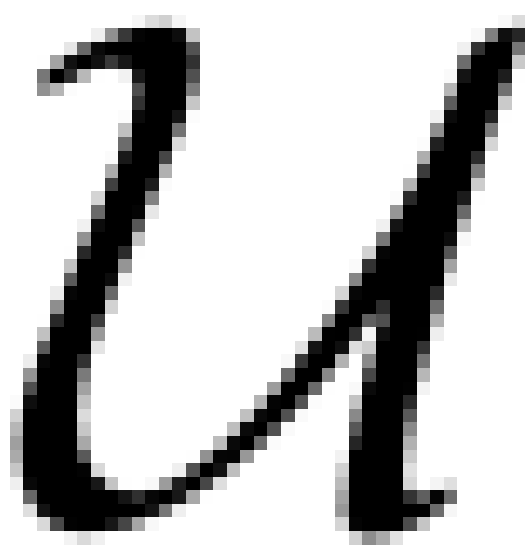

Fig. 4. Matrix U for UEs location

Based on this Lezi Update algorithm (with order-2 Markov Model), cumulative probability of UEs occurrence in each room is calculated and stored in a one-dimensional array (Say $C U[]$ ) of size R. It incorporates the probability of going to a particular room in next time slot conditioned on current location of the UE. The $i_{t h}$ entry of CU array represents the probability of finding any UE in room $i$. Finally, we start with deploying FAP at the room having with highest cumulative probability. Successively, deployment of FAPs is done until FAP count become equal to $R * P_{r}$.

\subsection{Adaptive Learning Based FAP detection}

Once FAPs are deployed, we need to analyze the statistics of FAP signal received at each room. For this, we maintain a $2 \mathrm{D}$ matrix (Say RoomFAP), where RoomFAP $[i][j]$ entry represents the number of times FAP $j$ 's signal is detected inside room $i$. Whenever a UE moves to room $i$, it tries to detect the signals from all FAPs in that room. If received signal from FAP $j$ is greater than the threshold than the value of $\operatorname{RoomFAP}[i][j]$ is incremented. This matrix is later used to calculate the probability of detecting different FAPs inside each room and for optimal resource reservation technique (Fig. 55.

\subsection{Expectation of UE call Arrival and Duration}

In dense femtocell network, reservation of resources helps in reducing UE call blocking, specially during inter-femtocell handover. For this, we suggest a prediction based resource reservation technique. This technique is based on Regression Model With ARIMA Time Series Errors which is often used for forecasting values based on historical data. This model explains the behavior of a model with predictor data, though the errors have autocorrelation indicative of an ARIMA process. The specific ARIMA model that is used is Box-Jenkins Model.

The Box-Jenkins methodology is a five-step process for identifying, selecting, and assessing conditional mean models (for discrete, univariate time series data). It involves following steps:

-Establishing the stationarity of given time series. If the series is not stationary, successive differencing of series is done to attain stationarity.

-When the model is stationary, we select a conditional mean model for our data. In our case, we have selected ARIMA model. It suggests differencing the non-stationary series one more time to achieve stationarity. Thus, ARIMA model combines both Auto-Regressive (AR) and Moving-Average (MA) models that were previously used, hence, it proves to be a better forecasting model.

- Specify the model and estimate the model parameters.

- Conduct goodness-of-fit checks to ensure the model describes your data adequately and check if the residuals are uncorrelated.

-Forecasting the future data

We use ARIMA model to predict inter-call arrival and call duration. For this, we input history of last $K$ inter-call arrival (or call duration) values to the model. After execution, model forecasts next expected inter-call arrival (or call duration). Finally, we use this forecasted inter-call arrival and call duration values for resource reservation in FAPs. Figure 6 explains the sequence of steps involved for forecasting a value using ARIMA model when a series

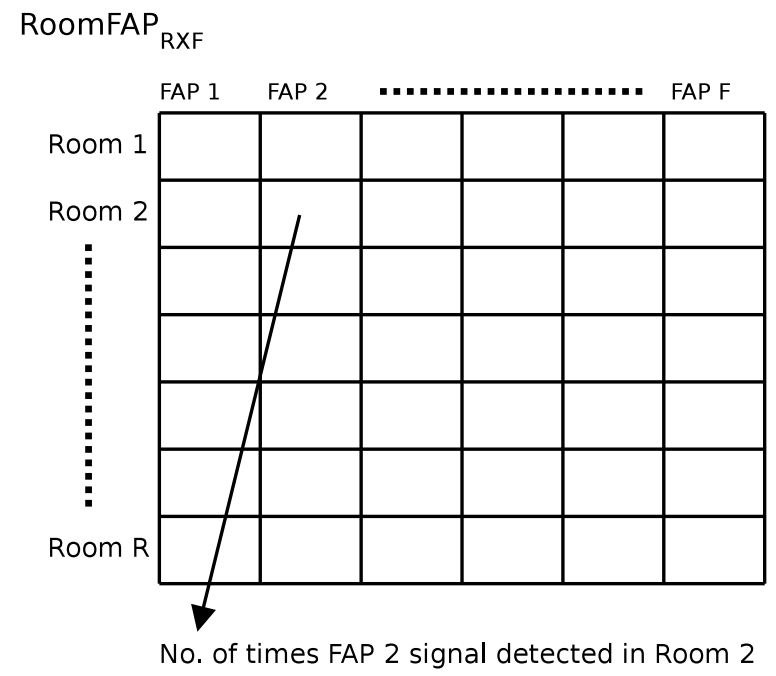

Fig. 5. Matrix $\mathcal{U}$ and array $C U$ for UEs location 


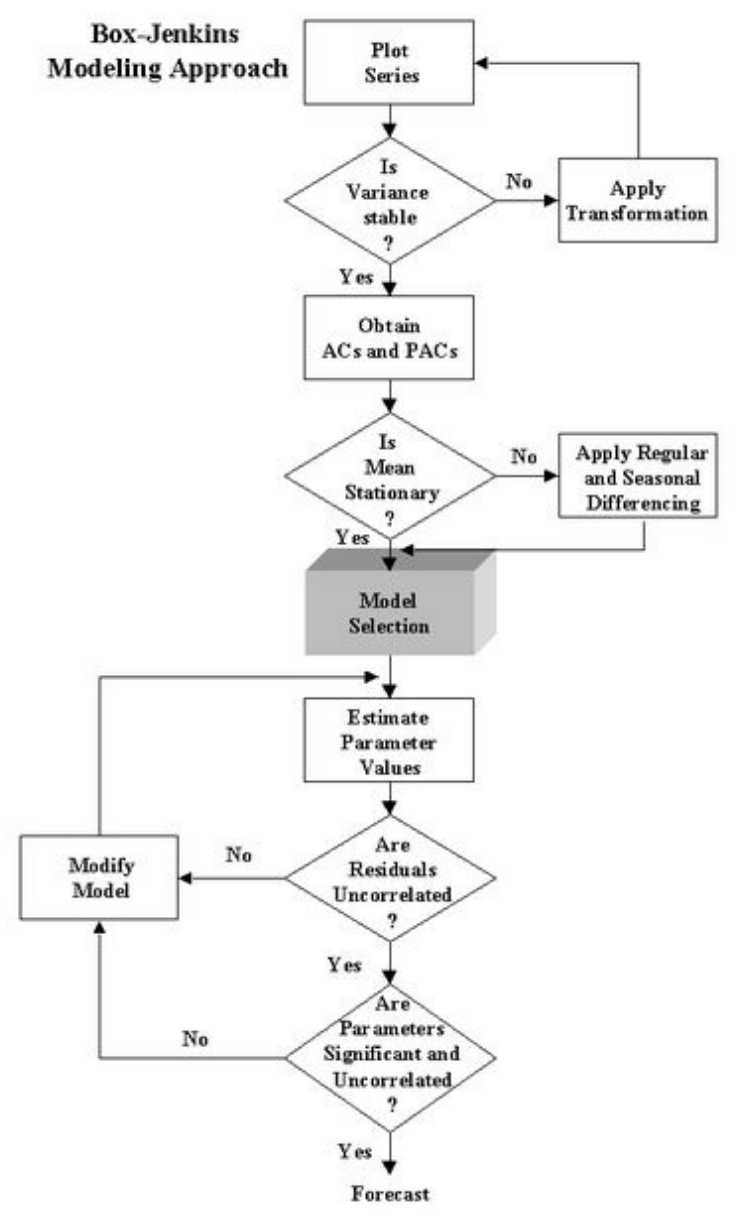

Fig. 6. Box-Jenkins Methodology

of values are given as input.

\subsection{Femtocell Resource Reservation}

Once we forecast the next inter-call arrival and call duration using ARIMA model, we need to reserve the resources at FAPs for future handovers. For each UE, we have to find the candidate FAPs for resource reservation. For this, we make use of two matrices $\mathcal{U}$ and RoomF AP as mentioned in previous subsections. When UE $i$ wants to reserve resources, it finds out a total of $\mathcal{F}$ candidate FAPs where UE is most probable to move. Candidate FAPs for UE $i$ selected based on the product of probability of UE $i$ in a room $j$ and probability of finding an FAP $k$ in that room $j$ as follows;

$$
\text { CandidateF } A P_{i}[\mathcal{F}]=\max _{k}\left\{\sum_{j=1}^{R} \mathcal{U}[i][j] * \operatorname{Room} F A P[j][k]\right\}
$$

where CandidateF $A P_{i}$ is the set of candidate FAPs where UE $i$ will reserve resource for future handover. Let Call $_{i}$ and Call $_{i}$ be the inter-call arrival and call duration forecast calculated through ARIMA model. Then, UE $i$ will reserve the resources needed for next $C$ all $A_{i}+$ Call $_{i}$ time slots in each FAP

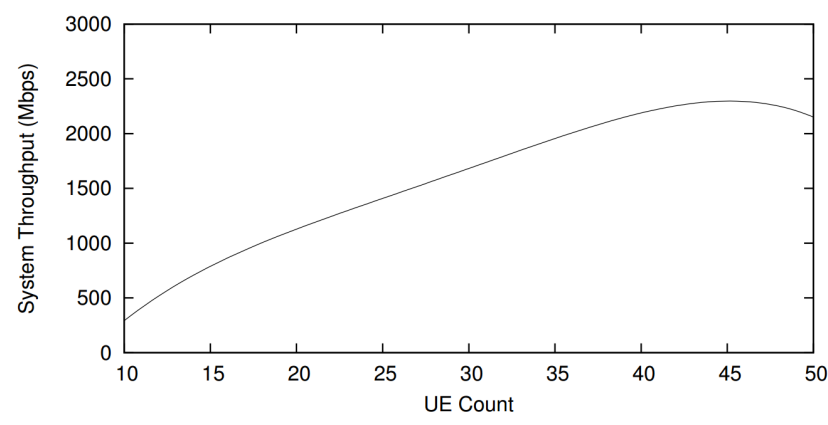

Fig. 7. UE count vs. System throughput

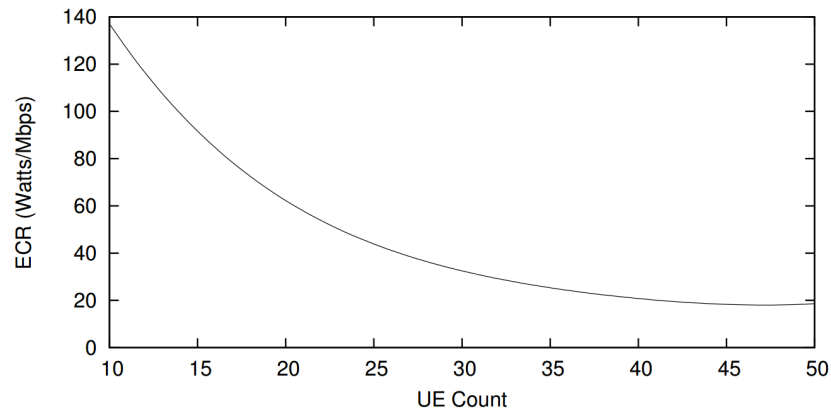

Fig. 8. UE count vs. ECR

$\in$ CandidateF $A P_{i}$. After this time duration, reservation for UE is cleared as to free up the resources for call arrivals from other UEs.

\section{SIMULATION RESULTS}

Our simulation model consists of a set of UEs distributed uniformly in the simulation region. FAPs are deployed at the center of the rooms, based on the given penetration ratio. Discrete event simulations are done where UEs call arrival and duration follows an exponential distribution with mean 600 and 180 seconds, respectively. All values are obtained for $95 \%$ confidence interval averaged over 100 iterations. The rest of the simulation parameters are summarized in Table I.

\begin{tabular}{|c|c|}
\hline Parameter & Value \\
\hline Bandwidth & $5 \mathrm{MHz}$ \\
\hline Number of Subchannels & 256 \\
\hline FAP Energy Consumption & $10 \mathrm{watt}$ \\
\hline FAP Penetration Ratio & $\{0.5-1.0\}$ \\
\hline UE Count & $\{10-50\}$ \\
\hline FAP Transmit Power $\left(P_{\max }\right)$ & $23 \mathrm{dBm}$ \\
\hline Gaussian Noise Figure & $-174 \mathrm{dBm} / \mathrm{Hz}$ \\
\hline Zero-Load FAP Power Consumption $\left(E_{0}\right)$ & $5 \mathrm{Watts}$ \\
\hline Path Loss Coefficient & 3.5 \\
\hline
\end{tabular}

To analyse the performance of our resource reservation technique, we calculate system throughput, energy efficiency and call blocking ratio for varying UE and FAP count. Figure 7 shows the effect of increasing UE count on overall system throughput. As can 


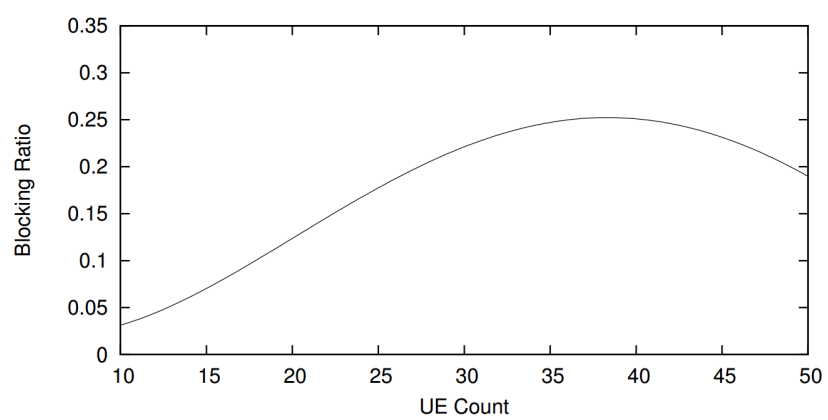

Fig. 9. UE count vs. Blocking Ratio

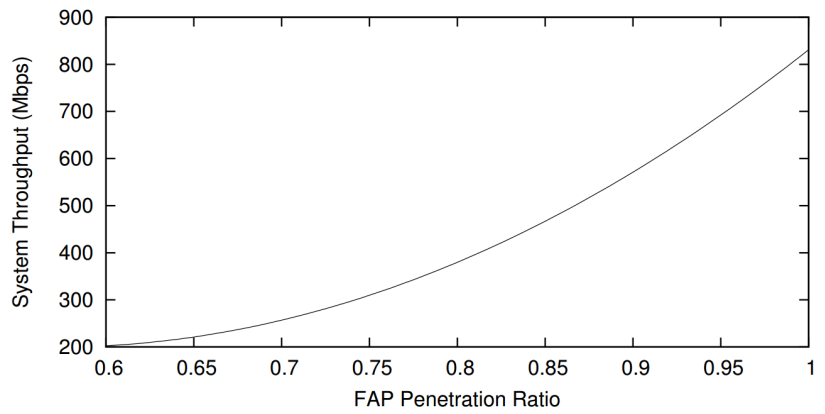

Fig. 10. System throughput vs. FAP penetration ratio

be seen that with an increase in UE count, more UE will be requesting for resources. Since FAPs are mostly underutilized, it can serve increased number of UEs thereby improving overall system throughput. However, with high UE count, we also see some decrease in system throughput. Figure 8 shows the effect of increasing UE count on ECR of the system. Since FAP count and their energy consumption remain constant, reduction in ECR is observed due to increase in system throughput. Talking about system blocking ratio (Fig. 9), we have seen an increase blocked users. This is due to the fact that higher successful reservation count implies a higher number of UEs making resource reservation requests at FAPs. This, in turn, reduces the resources available for other UEs thereby increasing the number of blocked requests too. Figure 10 shows the effect of increasing FAP penetration ratio on overall system throughput. As FAP penetration ratio increases, a number of rooms with FAP deployment also increases. With increase in FAP count, UE can detect more FAPs and hence reserve resources to them. This additionally reduces transmitter-receiver distance thereby improving SINR and bitrate. Figure 11 shows the effect of increasing FAP count on ECR of the system. With an increase in FAP count, system energy consumption also increases. However, a manifold increase in system throughput compensates for the higher energy consumption thereby reducing the ECR. Additionally, in Figure 12 we see a decrease in system blocking ratio with an increase in FAP penetration ratio because now more FAPs are available to serve users thereby reducing system blocking.

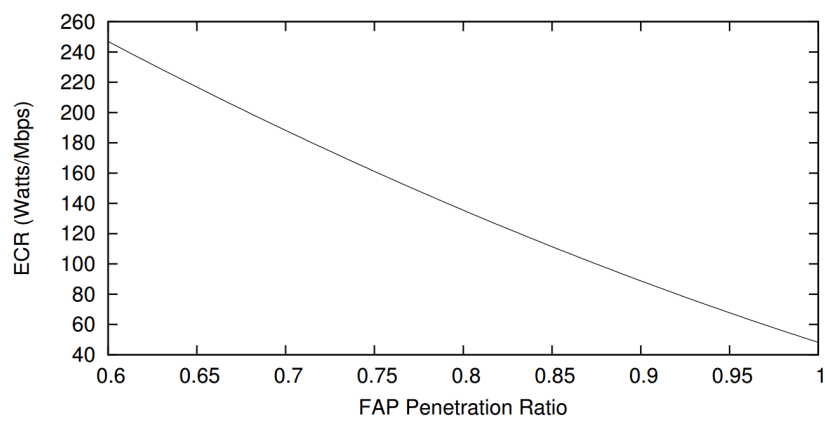

Fig. 11. ECR vs. FAP penetration ratio

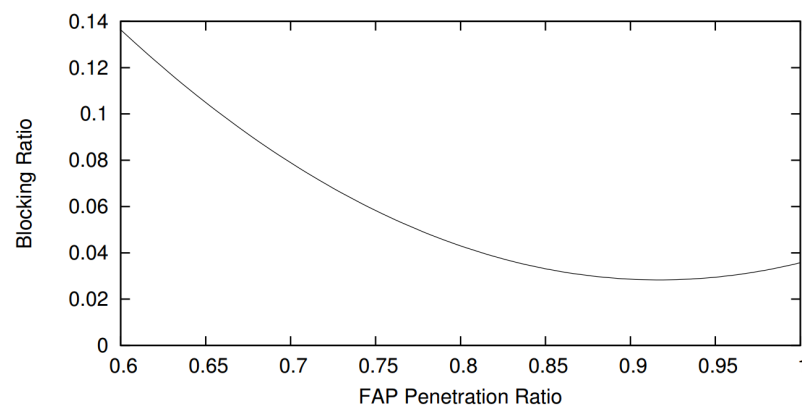

Fig. 12. Blocking ratio vs. FAP penetration ratio

\section{CONCLUSION}

In this paper, we have suggested an enhanced resource reservation technique for femtocell network with an aim to improve call blocking probability. On one hand, our suggested technique shows significant improvement in system throughput and energy efficiency, however, on the other hand, we have seen an increase in call blocking too. The mobile users who are able to successfully reserve resources in femtocells are able to maintain their connection during handover. However, reservation of resources on multiple femtocells results in availability of lower number of resources for other users. This consequently results in an increase in blocked connection requests for all possible femtocell and mobile user distributions. Our suggested technique occasionally shows quite an improvement in system blocking, however, it may not be optimal for all possible user deployments and mobility scenarios.

\section{REFERENCES}

[1] A. K. Vishwakarma, A. Jain, and S. Jain, "Present and future research directions for cell selection in femtocell networks," International Journal of Research in Technology, vol. 2, no. 2, pp. 5-10, April 2016.

[2] A. K. Sharma, P. N. Suman, and A. Jain, "Improving energy efficiency of femtocell network by joint estimation of downlink throughput and uplink power," in Proceedings of the International Conference on Advanced Computing and Communication Systems, Jan 2015, pp. 1-5.

[3] H. Abu-Ghazaleh and A. Alfa, "Mobility prediction and spatial-temporal traffic estimation in wireless networks," in 
Proceedings of the IEEE Vehicular Technology Conference, May 2008, pp. 2203-2207.

[4] J. Song, L. Li, and J. Han, "A fair scheduling algorithm combined with reservation resource compensation for wireless networks," in Proceedings of the Personal, Indoor and Mobile Radio Communications, vol. 3, Sept 2003, pp. 2338-2342.

[5] M. Chowdhury, S. H. Chae, and Y. M. Jang, "Group handover management in mobile femtocellular network deployment," in Proceedings of the Fourth International Conference on Ubiquitous and Future Networks, July 2012, pp. 162-165.

[6] N. Cai, J. Meng, W. Yan, Z. Bao, and P. Li, "Modeling and short-term forecasting of the electricity price based on fuzzy box-jenkins," in Proceedings of the 8th World Congress on Intelligent Control and Automation, July 2010, pp. 4619-4622.

[7] W. Tang, M. Wong, Y. Wong, and T. Chung, "Load forecasting by fuzzy neural network in box-jenkins models," in Proceedings of the IEEE International Conference on Systems, Man, and Cybernetics, vol. 2, Oct 1998, pp. 1738-1743.

[8] H. El Hag and S. Sharif, "An adjusted arima model for internet traffic," in Proceedings of the AFRICON, Sept 2007, pp. 1-6.

[9] S. Kang, S. Lee, Y. Won, and B. Seong, "On-line prediction of nonstationary variable-bit-rate video traffic," IEEE Trans- actions on Signal Processing, vol. 58, no. 3, pp. 1219-1237, March 2010

[10] B. Sklar, "Rayleigh fading channels in mobile digital communication systems .I. characterization," IEEE Communications Magazine, vol. 35, no. 7, pp. 90-100, July 1997.

[11] E. C. Cherry, "A history of the theory of information," Proceedings of the IEE - Part III: Radio and Communication Engineering, vol. 98, no. 55, pp. 383-393, Sept 1951.

[12] M. Deruyck, D. De Vulder, W. Joseph, and L. Martens, "Modelling the power consumption in femtocell networks," in Proceedings of the IEEE Wireless Communications and Networking Conference Workshops (WCNCW), Apr 2012, pp. 30-35.

[13] X. Wang, A. V. Vasilakos, M. Chen, Y. Liu, and T. T. Kwon, "A Survey of Green Mobile Networks: Opportunities and Challenges," ACM Mobile Networks and Applications, vol. 17, no. 1, pp. 4-20, Feb 2012.

[14] M. Z. Chowdhury and Y. M. Jang, "Handover management in highly dense femtocellular networks," EURASIP Journal on Wireless Communications and Networking, pp. 1-21, Jan 2013. 